\title{
Hyperglycemia and postoperative cognitive dys- function: another call for better glycemic control?
}

\author{
Hilary P. Grocott MD FRCPC FASE
}

W HEREAS research in the field of anesthesia has always fundamentally focused on optimizing the health and outcome of perioperative patients, it has distinctly evolved in recent decades from that principally directed at "anesthesia" per se (that is, anesthetic drugs and techniques, with their associated physiologic and other phenotypic responses), to that more related to broader perioperative issues. Such is the case for two clinical entities that frequently challenge the anesthesiologist: hyperglycemia and postoperative cognitive dysfunction (POCD). Postoperative cognitive dysfunction, although initially and extensively studied in the setting of cardiac surgery, ${ }^{1,2}$ is increasingly the focus of study in patients undergoing non-cardiac surgery. ${ }^{3,4}$ In a similar fashion, perioperative hyperglycemia, and its relationship to various clinical outcomes, has received considerable recent attention, both in non-cardiac and cardiac surgical populations. ${ }^{5,6}$ Though individually, these clinical entities are not new anesthesia research directions, their potential relationship to each other has recently received increased attention. ${ }^{6}$ Focusing on these perioperative entities represents a cogent example of how anesthesia research continues to move forward by turning attention inwards to some of the more subtle aspects of clinical care. The purpose of this editorial is to address both of these topics, not in extensive detail, but to direct attention to their commonality and relationship to each other.

Hyperglycemia is a frequent perioperative event with a variable incidence influenced both by patient characteristics (such as pre-existing diabetes) as well as type of surgical procedure (being more common during highly stressful operations such as cardiac surgery). Arguably, its relatively common perioperative occurrence is one reason why it is frequently under treated. Anesthesiologists may become desensitized and complacent to hyperglycemia when it is seen so regularly, and as well when it is not easily related to a recognizable adverse perioperative event. Another reason for a lack of enthusiasm for its treatment is the potential risk (significant hypoglycemia) associated with its over-treatment. ${ }^{7}$ The clinical significance of what most would consider relatively minor increases in perioperative glucose levels is likely underestimated. Indeed, defining the threshold as to what constitutes hyperglycemia is part of the problem as to why hyperglycemia is often inadequately addressed.

If one takes the most conservative definition of hyperglycemia (that is, a blood glucose just above the upper limit of normal), it is a relatively common entity during surgery. However, if one uses a more outcomes-based and disease-oriented approach to defining hyperglycemia, its incidence is somewhat lower, and the glucose threshold defining it variably higher. In essence, what is pertinent hyperglycemia for one outcome may be different than for another. For example, adverse outcome after head trauma has been demonstrated to increase when the glucose level is beyond a threshold of approximately $10 \mathrm{mmol} \cdot \mathrm{L}^{-1}$. For myocardial infarction, Suleiman et al. ${ }^{9}$ identified a fasting glucose level of $7.5 \mathrm{mmol} \cdot \mathrm{L}^{-1}$ as predictive of increased 30-day mortality. In stroke settings, a threshold as low as $7.0 \mathrm{mmol} \cdot \mathrm{L}^{-1}$ can increase the risk of adverse outcome. ${ }^{10}$ With an even lower glucose level defining hyperglycemia, considerable attention has been directed to intensive care unit (ICU) outcome with van den Berghe et $a .^{5}$ outlining increases in morbidity and mortality with glucose levels above $6 \mathrm{mmol} \cdot \mathrm{L}^{-1}$.

Recently, hyperglycemia has also been identified as a risk factor for POCD. Puskas et al., ${ }^{11}$ in a study of patients undergoing cardiac surgery utilizing cardiopulmonary bypass $(\mathrm{CPB})$, found hyperglycemia

CAN J ANESTH 2008/55: 3 / pp 140-145

From the Departments of Anesthesiology and Surgery, University of Manitoba, Winnipeg, Manitoba, Canada.

Address correspondence to: Dr. Hilary P. Grocott, University of Manitoba, I.H. Asper Clinical Research Institute, CR3008-369 Tache

Ave., Winnipeg, MB R2H 2A6, Canada. Phone: 204-258-1313; Fax: 204-231-0425; E-mail: hgrocott@sbgh.mb.ca 
(defined as a blood glucose $>11 \mathrm{mmol} \cdot \mathrm{L}^{-1}$ ) to occur in more than $75 \%$ of patients intraoperatively, with patients having pre-existing diabetes mellitus exhibiting an even higher incidence. Patients had cognitive function assessed both pre-and six weeks postoperatively with the incidence of POCD compared between those with and without intraoperative hyperglycemia. The hyperglycemic patients had a POCD incidence of $40 \%$ vs $29 \%$ in the normoglycemic group (odds ratio, $1.85,95 \%$ confidence intervals $1.1-3.0 ; P=0.0165)$. Hyperglycemia was the strongest factor associated with POCD in their multivariate analysis. This study used a very high threshold to define hyperglycemia, partly related to the fact that if a lower level had been used in this setting, so many patients would have met the definition for hyperglycemia that there would have been few normal non-hyperglycemic patients by which to compare the hyperglycemic patients to. There are several contributing factors for this high incidence of hyperglycemia in cardiac surgical patients, including the administration of glucose-containing solutions (cardioplegia and pump prime fluids), the stress responses inherent to surgery and $\mathrm{CPB}$ (with marked increases in circulating catecholamines), ${ }^{12}$ and hypothermia. ${ }^{13}$ Significant insulin antagonizing effects of the increased circulating catecholamines and cortisol, ${ }^{14}$ coupled with hypothermia during bypass, impairs the ability of insulin to transport glucose intracellularly. ${ }^{13}$ These data highlight a link between POCD and hyperglycemia and add to our understanding of the pathophysiology of POCD. It also serves to identify a potential opportunity for therapeutic intervention to reduce the incidence of POCD.

However, before moving directly to therapeutic opportunities, one must carefully examine the strength of this link first by asking if there is a biologically plausible reason why the two could be linked. Although the etiology and pathophysiology of POCD is not clearly defined, in cardiac surgery, there is considerable evidence that it may be related to cerebral ischemia (via global cerebral hypoperfusion or embolic phenomenon). ${ }^{15,16} \mathrm{~A}$ significant body of literature has addressed the relationship between hyperglycemia and adverse neurologic outcome. Experimental data outlining a causal relationship between hyperglycemia and adverse outcome after stroke is well established. ${ }^{17}$ From a pathophysiologic basis, elevated glucose levels allow a higher substrate availability for the production of lactate during the anaerobic metabolism consequent with cerebral ischemia. ${ }^{18,19}$ Intracellular lactic acidosis interferes with glycolysis, protein synthesis, and enzyme function, among other intracellular processes. $^{20}$ In addition, hyperglycemia has also been demonstrated during cerebral ischemia to increase the release of the excitotoxic amino acids glutamate and aspartate. These amino acids are key mediators in the ischemic cascade and hyperglycemia significantly augments this injurious response, a critical means by which cerebral outcome may be influenced. ${ }^{21,22}$ In addition, inflammatory pathways have been shown to be important in the pathophysiology of POCD. ${ }^{23}$ Hyperglycemia may also enhance the inflammatory response ${ }^{24}$ in one study, increasing perioperative Creactive protein levels. ${ }^{25}$ Additional hyperglycemiamediated inflammatory responses could augment inflammation-mediated POCD. Indeed, it is via both the ischemia and inflammatory pathways that there is a plausible biologic link between POCD and hyperglycemia.

Despite the emerging links between hyperglycemia and POCD, important limitations (and resulting inherent opportunities for research) exist in this literature. The best clinical studies in this field of research to date are observational and thus define only an association of hyperglycemia to POCD. Defining causation will require considerably more stringent further study. Indeed, some potential flaws in these links between hyperglycemia and other adverse outcomes have recently been demonstrated. ${ }^{26}$ This reinforces the concept of the difference between temporal association and causation, in essence, asking whether hyperglycemia can cause POCD, or if the two are only epiphenomena. One often incorrectly extrapolates data from such association studies assuming that addressing the associated factor will result in improvement of the adverse clinical outcome. Specifically, if hyperglycemia is associated with POCD, then correcting the hyperglycemia should reduce the incidence and severity of POCD. However, this needed type of prospective study, with glycemic control as the intervention and POCD as the outcome, has not yet been completed.

Even if the evidence linking hyperglycemia to POCD were more robust, there are significant limitations in effecting changes to perioperative glycemic levels. As common as it is, preventing hyperglycemia during cardiac surgery can be difficult. Frequently, even large doses of insulin fail to return glucose levels to normal during surgery. In addition, there is often some reluctance to aggressively treat hyperglycemia because of the risk of subsequent hypoglycemia. For example, in a study by Chaney et al., ${ }^{7}$ insulin administered during cardiac surgery increased the risk of post-bypass hypoglycemia. In addition, the study demonstrated considerable difficulty in actually attaining normoglycemia intraoperatively. Further safety 
concerns also exist related to cardiac dysrhythmias, as high-dose insulin can also result in significant hypokalemia due to its facilitation of potassium transmembrane transport mechanisms. An existing therapeutic limitation is the lack of rapid and reliable point-of-care blood glucose testing. To date, there are no userfriendly continuous glucose monitors - something definitely needed if truly aggressive insulin therapy is to be used.

Without doubt, both hyperglycemia and POCD will continue to challenge perioperative care for some time. At present, there is emerging evidence for a pathophysiologic, as well as a clinical link between hyperglycemia and POCD. However, significant gaps exist, making firm management recommendations difficult to offer. Perioperative hyperglycemia is clearly an important finding. However, it is uncertain at what blood glucose level concern should be raised, and what therapy is appropriate. Common sense dictates that normoglycemia should be the goal, but inadvertent hypoglycemia could be just as injurious. Important opportunities for further research exist in both these perioperative fields, corroborating a hyperglycemia-POCD link, and identifying effective and safe therapeutic strategies.

\section{L'hyperglycémie et la dysfonction cognitive postopératoire : faut-il un meilleur contrôle de la glycémie ?}

Alors que la recherche en anesthésie s'est traditionnellement concentrée essentiellement sur des questions d'optimisation de la santé et du devenir des patients en période périopératoire, elle a évolué de façon distincte ces dernières décennies d'une recherche orientée principalement vers l'anesthésie en soi (c'est-à-dire, les techniques et médicaments anesthésiques, ainsi que les réactions physiologiques et phénotypiques associées) vers une recherche traitant de questions périopératoires plus vastes. En effet, c'est le cas de deux entités cliniques qui posent régulièrement des problèmes à l'anesthésiologiste : l'hyperglycémie et la dysfonction cognitive postopératoire (DCPO). La dysfonction cognitive postopératoire, à l'origine très étudiée dans le contexte d'une population de chirurgie cardiaque, ${ }^{1,2}$ constitue de plus en plus un champ d'étude chez les patients subissant des chirurgies non cardiaques. ${ }^{3,4}$ De même, l'hyperglycémie périopératoire et sa relation à diverses évolutions cliniques font récemment l'objet d'une attention considérable aussi bien chez les patients de chirurgie cardiaque que non cardiaque. ${ }^{5,6}$ Lorsque pris à part, ces entités cliniques ne constituent aucunement de nouvelles orientations de recherche en anesthésie ; néanmoins, la relation potentielle qu'elles entretiennent a récemment reçu une attention grandissante. ${ }^{6}$ Le simple fait que la recherche se concentre actuellement sur ces entités périopératoires représente une preuve irréfutable de la manière dont la recherche en anesthésie continue de progresser : celle-ci concentre désormais son attention sur des questions au cœur même de la pratique, en se tournant vers certains des aspects plus subtils des soins cliniques. L'objectif de cet éditorial est de rendre compte de ces deux sujets, non pas de manière exhaustive, mais plutôt en attirant l'attention sur leur points communs et leur relation.

L'hyperglycémie est un événement périopératoire fréquent présentant une incidence variable, laquelle est influencée par les caractéristiques du patient (comme par exemple un diabète préexistant) ainsi que par le type d'intervention chirurgicale (elle est plus répandue lors d'opérations provoquant un niveau élevé de stress, comme par exemple lors de chirurgie cardiaque). C'est sans doute parce qu'elle survient assez fréquemment en période périopératoire qu'elle est souvent sous-traitée. Les anesthésiologistes pourraient devenir indifférents et passifs face à l'hyperglycémie étant donné qu'elle apparaît si régulièrement, en plus du fait qu'elle n'est pas facilement associée à un événement périopératoire indésirable identifiable. Une autre raison qui pourrait expliquer le peu d'enthousiasme à traiter l'hyperglycémie réside dans le risque potentiel (hypoglycémie significative) associé à un traitement excessif.7 L'importance clinique de ce que la plupart des anesthésiologistes ne considéreraient que comme des augmentations relativement faibles des niveaux de glucose périopératoire est probablement sous-estimée. En effet, la définition même du seuil au-delà duquel on parle d'hyperglycémie est une partie inhérente du problème, et constitue l'une des raisons pour lesquelles l'hyperglycémie est souvent abordée de façon inappropriée.

Si l'on prend la définition plus traditionnelle de l'hyperglycémie (c'est-à-dire, un glucose sanguin juste au-dessus de la limite supérieure de ce qui est considéré normal), il s'agit d'une entité relativement fréquente pendant une chirurgie. Toutefois, lorsqu'on s'appuie 
sur une approche davantage orientée sur les devenirs et les maladies pour définir l'hyperglycémie, son incidence est un peu plus faible, et le seuil de glucose la définissant variablement plus élevé. En fait, une hyperglycémie jugée pertinente pour un devenir peut être différente pour un autre. Par exemple, il a été démontré que les effets indésirables à la suite d'un traumatisme crânien augmentent lorsque la glycémie est supérieure à un seuil d'environ $10 \mathrm{mmol} \cdot \mathrm{L}^{-1}{ }^{8}$ Dans les cas d'infarctus du myocarde, Suleiman et coll. ${ }^{9}$ ont démontré qu'une glycémie à jeun de $7,5 \mathrm{mmol} \cdot \mathrm{L}^{-1}$ constituait un prédicteur de mortalité à 30 jours. Dans des contextes d'accident vasculaire cérébral, un seuil de 7,0 $\mathrm{mmol} \cdot \mathrm{L}^{-1}$ seulement peut augmenter les risques d'événements indésirables. ${ }^{10}$ Sur la base d'un niveau glycémique plus bas encore pour définir l'hyperglycémie, une grande attention a été portée sur les devenirs en soins intensifs (ICU) lorsque van den Berghe et coll. ${ }^{5}$ ont rapporté des taux de mortalité et de morbidité plus élevés lors de glycémie au-dessus de $6 \mathrm{mmol} \cdot \mathrm{L}^{-1}$.

En outre, l'hyperglycémie a récemment été identifiée comme un facteur de risque pour la DCPO. Puskas et coll., ${ }^{11}$ dans une étude sur des patients subissant une chirurgie cardiaque utilisant la circulation extra-corporelle (CEC), ont découvert que l'hyperglycémie (définie en tant que glucose sanguin $>11 \mathrm{mmol} \cdot \mathrm{L}^{-1}$ ) survenait chez plus de $75 \%$ des patients pendant l'opération, les patients ayant un diabète préexistant présentant une incidence encore plus élevée. Les patients ont fait l'objet d'une évaluation de la fonction cognitive lors d'un bilan préopératoire et six semaines après l'opération; les auteurs ont ensuite comparé l'incidence de DCPO entre ces deux évaluations chez les sujets avec et sans hyperglycémie peropératoire. Les patients hyperglycémiques ont montré une incidence de DCPO de $40 \%$ comparée à $29 \%$ dans le groupe normoglycémique (rapport de cotes, $1,85,95 \%$ intervalle de confiance $1,1-3,0 ; P=0,0165)$. Dans l'analyse multifactorielle des auteurs, l'hyperglycémie a constitué le facteur associé le plus fortement à la DCPO. Cette étude a utilisé un seuil très élevé pour définir l'hyperglycémie, en partie en raison du fait que si un seuil plus bas avait été déterminé dans ce contexte, un nombre tellement élevé de patients aurait rempli les critères de définition de l'hyperglycémie qu'il n'y aurait eu que peu de patients normaux non hyperglycémiques auxquels comparer les patients hyperglycémiques. Il existe de nombreux facteurs contribuant à l'incidence élevée d'hyperglycémie chez les patients subissant une chirurgie cardiaque, dont l'administration de solutions contenant du glucose (liquides de cardioplégie et d'amorçage de la pompe), les réactions de stress inhérentes à la chirurgie et la CEC (avec des augmentations marquées des catécholamines circulantes), ${ }^{12}$ et l'hypothermie. ${ }^{13}$ Les effets antagonistes importants des catécholamines circulantes et du cortisol sur l'insuline $^{14}$ associés à une CEC hypothermique mitigent la capacité de l'insuline à transporter le glucose à l'intérieur des cellules. ${ }^{13}$ Ces données soulignent un lien entre la DCPO et l'hyperglycémie et ajoutent à notre compréhension de la physiopathologie de la DCPO. Elles contribuent également à l'identification d'interventions thérapeutiques possibles afin de réduire l'incidence de la DCPO.

Cependant, avant de traiter des possibilités thérapeutiques, il faut examiner en détail la force du lien entre l'hyperglycémie et la DCPO, tout d'abord en se demandant s'il existe une explication biologique plausible à l'association de ces deux entités. En chirurgie cardiaque, bien que l'étiologie et la physiopathologie de la DCPO ne soient pas clairement définies, de nombreuses données indiquent qu'elle pourrait être associée à l'ischémie cérébrale (via une hypoperfusion cérébrale globale ou un phénomène embolique). ${ }^{15,16}$ Un corpus considérable de littérature traite de la relation entre l'hyperglycémie et le devenir neurologique indésirable. Des données expérimentales exposant une relation causale entre l'hyperglycémie et les événements indésirables après un AVC sont bien établies. ${ }^{17}$ D'un point de vue physiopathologique, les niveaux élevés de glucose permettent une disponibilité des substrats plus élevée pour la production de lactate pendant un métabolisme anaérobique suite à une ischémie cérébrale. ${ }^{18,19}$ L'acidose lactique intracellulaire interfère avec la glycolyse, la synthèse des protéines et la fonction enzymatique, parmi d'autres processus intracellulaires. ${ }^{20}$ En outre, il a également été démontré que l'hyperglycémie facilite la libération des acides aminés excitotoxiques glutamate et aspartate pendant l'ischémie cérébrale. Ces acides aminés sont des intermédiaires clés dans la cascade ischémique et l'hyperglycémie aggrave significativement cette réaction nocive, un moyen crucial qui peut influencer le devenir cérébral. ${ }^{21,22}$ De plus, il a aussi été montré que les voies inflammatoires jouent un rôle important dans la physiopathologie de la DCPO. ${ }^{23}$ L'hyperglycémie pourrait également augmenter la réaction inflammatoire ${ }^{24}$ et, dans une étude, a augmenté les niveaux de protéine $\mathrm{C}$ réactive périopératoires. ${ }^{25}$ D’autres réactions inflammatoires causées par l'hyperglycémie pourraient augmenter la DCPO provoquées par une inflammation. En effet, c'est au travers de l'ischémie et des voies inflammatoires qu'il existe un lien biologique plausible entre la DCPO et l'hyperglycémie.

Malgré les liens mis au jour entre l'hyperglycémie 
et la DCPO, d'importantes limites (et les opportunités de recherche inhérentes en résultant) existent dans ce corpus de littérature. Les meilleures études cliniques dans ce domaine de recherche sont observationnelles et ne définissent dès lors qu'une association de l'hyperglycémie à la DCPO. La définition du lien de causalité nécessitera des études plus poussées et bien plus rigoureuses. En effet, des failles potentielles des liens établis entre l'hyperglycémie et d'autres devenirs néfastes ont récemment été démontrées. ${ }^{26} \mathrm{Ceci}$ renforce le concept d'une différence entre une association temporelle et un lien de causalité, en d'autres termes, entre se demander si l'hyperglycémie peut provoquer une DCPO ou si les deux ne constituent que des épiphénomènes. Il arrive souvent que l'on extrapole des données d'études d'associations telles que celles-ci en présumant que la prise en considération du facteur associé engendrera une amélioration du devenir clinique négatif. Plus spécifiquement, si l'hyperglycémie est associée à la DCPO, la correction de l'hyperglycémie devrait dès lors réduire l'incidence et la sévérité de la DCPO. Toutefois, une telle étude prospective, prenant le contrôle glycémique comme intervention et la DCPO comme résultat, bien que nécessaire, n’a pas encore été menée.

Même si les preuves associant l'hyperglycémie à la DCPO étaient plus solides, des limites considérables existent quand on tente d'apporter des changements à la glycémie en période périopératoire. Aussi fréquente que soit l'hyperglycémie, la prévenir durant une chirurgie cardiaque peut néanmoins être un défi. Il est courant que même des doses importantes d'insuline ne parviennent pas à rétablir des niveaux glycémiques normaux pendant une chirurgie. En outre, on rencontre souvent une certaine résistance à traiter agressivement l'hyperglycémie en raison du risque subséquent d'hypoglycémie. Par exemple, dans une étude de Chaney et coll., ${ }^{7}$ l'insuline administrée pendant une chirurgie cardiaque a augmenté le risque d'hypoglycémie postCEC. De plus, l'étude a démontré d'importantes difficultés à atteindre véritablement une normoglycémie pendant l'opération. Il y a aussi d'autres questions de sécurité associées aux dysrythmies cardiaques, étant donné que l'insuline à haute dose peut également avoir pour résultat une hypokaliémie importante en raison de son effet de facilitation des mécanismes de transport du potassium à travers les membranes. Une des limites thérapeutiques existante est l'absence de glucomètre de proximité rapide et fiable. À ce jour, il n'existe pas de glucomètre continu facile d'emploi, ce qui serait absolument nécessaire si l'on voulait pratiquer une thérapie à l'insuline véritablement agressive.

Il est certain que l'hyperglycémie et la DCPO continueront de poser problème dans le contexte des soins périopératoires pour un temps. À l'heure actuelle, de plus en plus de données apparaissent soutenant un lien physiopathologique aussi bien que clinique entre l'hyperglycémie et la DCPO. Toutefois, des lacunes considérables demeurent, et il est dès lors malaisé d'offrir des recommandations de prise en charge robustes. L'hyperglycémie périopératoire constitue clairement une découverte importante. Néanmoins, il est encore difficile de déterminer à quel niveau de glycémie on devrait s'inquiéter, et par conséquent, quel est le traitement adéquat. Le bon sens voudrait que la normoglycémie soit l'objectif, mais une hypoglycémie malencontreuse pourrait être tout aussi nocive. Nombre d'opportunités importantes de recherche plus approfondie existent dans ces deux domaines de la médecine périopératoire que sont l'établissement d'un lien entre l'hyperglycémie et la dysfonction cérébrale postopératoire et l'identification de stratégies de traitement sécuritaires et efficaces.

\section{References}

1 Djaiani G, Fedorko L, Borger MA, et al. Continuousflow cell saver reduces cognitive decline in elderly patients after coronary bypass surgery. Circulation 2007; 116: 1888-95.

2 Newman MF, Kirchner JL, Phillips-Bute B, et al. Longitudinal assessment of neurocognitive function after coronary-artery bypass surgery. N Engl J Med 2001; 344: 395-402.

3 Monk TG, Weldon BC, Garvan CW, et al. Predictors of cognitive dysfunction after major noncardiac surgery. Anesthesiology 2008; 108: 18-30.

4 Maze M, Cibelli M, Grocott HP. Taking the lead in research into postoperative cognitive dysfunction. Anesthesiology 2008; 108: 1-2.

5 van den Berghe $G$, Wouters $P$, Weekers $F$, et al. Intensive insulin therapy in the critically ill patients. N Engl J Med 2001; 345: 1359-67.

6 Puskas F, Grocott HP, White WD, Mathew JP, Newman $M F$, Bar-Yosef $S$. Intraoperative hyperglycemia and $\operatorname{cog}$ nitive decline after CABG. Ann Thorac Surg 2007; 84: 1467-73.

7 Chaney MA, Nikolov MP, Blakeman BP, Bakhos M. Attempting to maintain normoglycemia during cardiopulmonary bypass with insulin may initiate postoperative hypoglycemia. Anesth Analg 1999; 89: 1091-5.

8 Lam AM, Winn HR, Cullen BF, Sundling N. Hyperglycemia and neurological outcome in patients with head injury. J Neurosurg 1991; 75: 545-51

9 Suleiman $M$, Hammerman H, Boulos $M$, et al. Fasting glucose is an important independent risk factor for 30-day mortality in patients with acute myocardial 
infarction: a prospective study. Circulation 2005; 111 : 754-60.

10 Alvarez-Sabin J, Molina CA, Ribo M, et al. Impact of admission hyperglycemia on stroke outcome after thrombolysis: risk stratification in relation to time to reperfusion. Stroke 2004; 35: 2493-8.

11 Puskas F, Grocott HP, White WD, Mathew JP, Newman $M F$, Bar-Yosef $S$. Intraoperative hyperglycemia and cognitive decline after CABG. Ann Thorac Surg 2007; 84: 1467-73.

12 Reves JG, Karp RB, Buttner EE, et al. Neuronal and adrenomedullary catecholamine release in response to cardiopulmonary bypass in man. Circulation 1982; 66: 49-55.

13 Lehot JJ, Piriz H, Villard J, Cohen R, Guidollet J. Glucose homeostasis. Comparison between hypothermic and normothermic cardiopulmonary bypass. Chest 1992; 102: 106-11.

14 McCowen KC, Malhotra A, Bistrian BR. Stress-induced hyperglycemia. Crit Care Clin 2001; 17: 107-24.

15 Mutch WA, Ryner LN, Kozlowski P, et al. Cerebral hypoxia during cardiopulmonary bypass: a magnetic resonance imaging study. Ann Thorac Surg 1997; 64: 695-701.

16 Pugsley W, Klinger L, Paschalis C, Treasure T, Harrison $M$, Newman $S$. The impact of microemboli during cardiopulmonary bypass on neuropsychological functioning. Stroke 1994; 25: 1393-9.

17 Wass CT, Lanier WL. Glucose modulation of ischemic brain injury: review and clinical recommendations. Mayo Clin Proc 1996; 71: 801-12.

18 Siesjo BK, Katsura KI, Kristian T, Li PA, Siesjo P. Molecular mechanisms of acidosis-mediated damage. Acta Neurochir Suppl 1996; 66: 8-14.

19 Vannucci RC, Brucklacher RM, Vannucci SJ. The effect of hyperglycemia on cerebral metabolism during hypoxia-ischemia in the immature rat. J Cereb Blood Flow Metab 1996; 16: 1026-33.

20 Nedergaard M, Goldman SA, Desai S, Pulsinelli WA. Acid-induced death in neurons and glia. J Neurosci 1991; 11: 2489-97.

21 Li PA, Shuaib A, Miyashita H, He QP, Siesjo BK, Warner DS. Hyperglycemia enhances extracellular glutamate accumulation in rats subjected to forebrain ischemia. Stroke 2000; 31: 183-92.

22 Rejdak K, Rejdak R, Sieklucka-Dziuba M, Stelmasiak $Z$, Grieb P. The effects of citicoline and/or MK-801 on survival, neurological and behavioral outcome of mice exposed to transient hyperglycemia and oligemic hypoxia. Eur Neuropsychopharmacol 2001; 11: 33341.

23 Mathew JP, Podgoreanu MV, Grocott HP, et al. Genetic variants in $\mathrm{P}$-selectin and $\mathrm{C}$-reactive protein influence susceptibility to cognitive decline after cardiac surgery. J Am Coll Cardiol 2007; 49: 1934-42.

24 Kinoshita K, Kraydieh S, Alonso O, Hayashi N, Dietrich $W D$. Effect of posttraumatic hyperglycemia on contusion volume and neutrophil accumulation after moderate fluid-percussion brain injury in rats. J Neurotrauma 2002; 19: 681-92.

25 Visser L, Zuurbier CJ, Hoek FJ, et al. Glucose, insulin and potassium applied as perioperative hyperinsulinaemic normoglycaemic clamp: effects on inflammatory response during coronary artery surgery. Br J Anaesth 2005; 95: 448-57.

26 Gandhi GY, Nuttall GA, Abel MD, et al. Intensive intraoperative insulin therapy versus conventional glucose management during cardiac surgery: a randomized trial. Ann Intern Med 2007; 146: 233-43. 